Vinayak Nagaraja, MBBS, MS, MMed (Clin Epi), FRACP

Department of Cardiovascular Medicine, Cleveland Clinic
A. Michael Lincoff, MD

Vice-Chairman, Department of Cardiovascular Medicine,

Cleveland Clinic; Professor, Cleveland Clinic Lerner College

of Medicine of Case Western Reserve University, Cleveland, $\mathrm{OH}$

\title{
Stable coronary artery disease: Intervene or not?
}

W HEN THE International Study of Comparative Health Effectiveness With Medical and Invasive Approaches (ISCHEMIA) trial $^{1-3}$ was presented at the American Heart Association meeting in November, 2019, it generated multiple headlines in major news sources. CNN covered the story by saying, "For heart disease, meds may work as well as invasive surgery, major trial shows." 4 The Washington Post published a story entitled "Stents and bypass surgery are no more effective than drugs for stable heart disease, highly anticipated trial results show."

Stable coronary artery disease needs a patientcentered approach; one size does not fit all
See related article, page 401

Neither these nor other similar headlines accurately convey the current body of evidence regarding the role of coronary revascularization in patients with coronary artery disease. The benefits of early revascularization in reducing mortality and reinfarction risk have been well established in patients who have unstable angina at rest, acute myocardial infarction, and significant unprotected left main coronary artery disease. ${ }^{6-9}$ However, since the publication of the Clinical Outcomes Utilizing Revascularization and Aggressive Drug Evaluation (COURAGE) trial ${ }^{10}$ in 2007, we have also known that patients with stable angina or ischemia do not experience a reduction in the risk of death, myocardial infarction, or cardiac arrest with percutaneous coronary intervention. Importantly though, percutaneous coronary intervention is associated with better relief of ischemic symptoms and quality of life than medical therapy alone.

Here, we describe the design, results, and limitations of the recent ISCHEMIA trial ${ }^{1-3}$ doi:10.3949/ccjm.87a.20048 and how its findings confirm and expand our existing understanding of the role of coronary revascularization in patients with stable ischemic syndromes.

\section{ISCHEMIA TRIAL DESIGN AND METHODOLOGY}

The ISCHEMIA trial ${ }^{1-3}$ was a randomized trial that compared, in a 1-to-1 ratio, an initial invasive or conservative treatment strategy in 5,179 patients with stable coronary artery disease with moderate or severe ischemia by noninvasive stress testing.

The key exclusion criteria were:

- Significant unprotected left main coronary artery disease ( $\geq 50 \%$ stenosis)

- Estimated glomerular filtration rate less than $30 \mathrm{~mL} / \mathrm{min} / 1.73 \mathrm{~m}^{2}$

- New York Heart Association class III or IV heart failure

- Recent myocardial infarction, percutaneous coronary intervention, or coronary artery bypass grafting

- Severe left ventricular dysfunction (ejection fraction $<35 \%$ )

- Intolerable angina at baseline.

All patients received guideline-directed optimal medical therapy. Coronary computed tomography was performed to exclude left main disease unless the coronary anatomy was previously defined. Patients with renal dysfunction did not undergo coronary computed tomography. The invasive strategy group underwent coronary angiography followed by revascularization within 1 month after randomization.

The primary end point of the trial was a composite of cardiovascular death, nonfatal myocardial infarction, hospitalization for unstable angina, hospitalization for heart failure, 
or resuscitated cardiac arrest. The key secondary end points in North American participants were a composite of cardiovascular death and nonfatal myocardial infarction, angina, and measures of quality of life and health economics.

Concurrent studies were performed in patients with advanced chronic renal failure ${ }^{11}$ and nonobstructive coronary artery disease with inducible ischemia.

\section{TRIAL RESULTS}

It took over a decade for the trial investigators to enroll 8,518 patients after screening nearly 26,000 candidates.

Of these 8,518 patients, 3,339 were excluded for various reasons, eg:

- 1,350 did not have moderate or severe ischemia on stress testing

- 1,218 did not have obstructive coronary artery disease

- 434 had left main coronary artery disease.

Coronary computed tomography was performed in $73 \%$ of patients.

Most $(77 \%)$ of the patients were men, and the median age was 64 . About $40 \%$ had diabetes mellitus. Most (90\%) had a history of stable angina, and 29\% had progressive angina in the previous 3 months, while $34.3 \%$ had no angina in the previous 4 weeks. Moderate to severe ischemia was documented in $87 \%$. Nearly everyone in the trial was receiving a statin and aspirin, nearly $60 \%$ had a low-density lipoprotein cholesterol level less than 70 $\mathrm{mg} / \mathrm{dL}$, and $77 \%$ had a systolic blood pressure lower than $140 \mathrm{~mm} \mathrm{Hg}$.

Among patients randomized to the invasive strategy, approximately $80 \%$ underwent revascularization, of whom $25 \%$ underwent surgical revascularization (using the internal mammary artery in 92\%). Most of the percutaneous coronary interventions were performed using second-generation drug-eluting stents $(\mathrm{n}=1,329)$.

In the conservative strategy group, $28 \%$ of the patients ultimately underwent cardiac catheterization and revascularization for suspected or confirmed events (13.8\%), optimal medical therapy failure $(3.9 \%)$, or nonadherence $(8.1 \%)$.

In the invasive strategy group, conventional coronary angiography confirmed triple- vessel disease ( $\geq 50 \%$ stenosis on quantitative coronary angiography) in $39.6 \%$ of the patients. More than one-third of the patients had proximal left anterior descending lesions ( $\geq 50 \%$ stenosis on quantitative coronary angiography). Fractional flow reserve-guided revascularization was performed in $20.3 \%$ of the cases. More than 400 patients in the invasive-strategy group underwent no revascularization, as a large proportion (221) of them had nonobstructive coronary artery disease. Coronary anatomy was found to be unsuitable for revascularization in 111 patients, and 28 patients preferred not to undergo revascularization.

\section{Outcomes}

After a median follow-up of 3.2 years, the event rates in the invasive vs conservative strategy groups did not differ significantly for the primary outcome (hazard ratio [HR] 0.93, 95\% confidence interval [CI] $0.80-1.08$ ) or cardiovascular death or myocardial infarction (HR 0.90, 95\% CI 0.77-1.06).

However, the rate of spontaneous myocardial infarction was lower in the invasive strategy group (HR 0.67, 95\% CI 0.53-0.83), as was the rate of hospitalization for unstable angina (HR 0.50, 95\% CI 0.27-90). The rate of periprocedural myocardial infarction was higher in the percutaneous coronary intervention group than in the medical therapy group (HR $2.98,95 \%$ CI 1.87-4.74), although periprocedural myocardial infarctions have been shown to have less prognostic importance (unless associated with $Q$ waves or creatine kinase-MB $>10$ times the upper limit of normal) than spontaneous myocardial infarctions. ${ }^{12}$

This finding raises the intriguing possibility of the benefit of revascularization in reducing spontaneous myocardial infarctions beyond symptom improvement in this patient population. However, spontaneous myocardial infarction was not a prespecified end point and thus must be regarded as a hypothesis-generating result that requires further validation. Moreover, the greater incidence of spontaneous myocardial infarction did not affect overall mortality, at least over the median 3.2 years of follow-up. Long-term follow-up might shed more light on the impact of spontaneous myocardial infarction on mortality and whether

\section{More than} a decade was needed to enroll 8,518 patients, after screening nearly $\mathbf{2 6 , 0 0 0}$ candidates 
the rates of myocardial infarction continue to diverge between the 2 treatment groups over time. It would also be interesting to see the characteristics of the spontaneous myocardial infarction in each cohort, especially if they were large or complicated.

\section{Results in patients with chronic kidney disease}

The ISCHEMIA-CKD trial ${ }^{11,13}$ assessed the same hypothesis as the ISCHEMIA trial in 777 patients with renal dysfunction (endstage renal disease on dialysis or estimated glomerular filtration rate less than $30 \mathrm{~mL} /$ $\min / 1.73 \mathrm{~m}^{2}$ ). The findings were similar to those of the ISCHEMIA trial, although the incidence of stroke was higher with the invasive strategy (HR 3.76, 95\% CI 1.52-9.32), as was the rate of death or new dialysis (HR 1.48, 95\% CI 1.04-2.11). Interestingly, the invasive approach was beneficial in individuals with severe inducible ischemia.

\section{Quality of life}

The burden associated with medical therapy and recurrent symptoms is not inconsequential. Frequent trips to outpatient clinics and

The impact of complete revascularization, the mode of revascularization, and invasive functional assessment are yet to be explored in the ISCHEMIA trial emergency departments for recurrent symptoms and inability to participate in cardiac rehabilitation and exercise and weight-loss programs impair quality of life in patients with coronary artery disease. ${ }^{14}$ Multiple previous trials $^{10,15}$ have shown improvement in quality of life after percutaneous coronary intervention in patients with stable angina.

In the ISCHEMIA trial, ${ }^{3}$ patients randomized to invasive therapy had significantly greater improvements in disease-specific health status (angina symptoms, functional status, and quality of life) than those in the conservative treatment group.

While differences in health status were only modest in the overall trial population, this was driven by the fact that $35 \%$ did not have angina at baseline, and $44 \%$ had angina only 1 to 3 times per month. A minority of the trial population, only $20 \%$, were severely symptomatic with daily or weekly angina. Not surprisingly, patients who were asymptomatic or minimally symptomatic at baseline had little or no change in symptom status over follow-up. In contrast, patients with moderate to severe symptoms at baseline had substan- tial improvements in angina frequency and quality of life with revascularization compared with conservative management, benefits that were sustained over the 36-month observation period. Similar findings were observed in the ISCHEMIA-CKD trial. ${ }^{3,16}$

\section{LIMITATIONS OF THE ISCHEMIA TRIAL}

Due to slow recruitment and lower-thanexpected event rates, the primary end point was changed from cardiovascular death or nonfatal myocardial infarction to a composite of cardiovascular death, nonfatal myocardial infarction, hospitalization for unstable angina, hospitalization for heart failure, or resuscitated cardiac arrest. Although this modification was a prespecified contingency and was enacted before the trial was unblinded, it raises questions regarding whether additional end points like resuscitated cardiac arrest or heart failure are relevant to percutaneous coronary intervention in stable coronary artery disease.

More relevant to the interpretability of the trial was that patients with lower ischemia burden $(5 \%)$ and abnormal exercise tolerance testing were included to increase enrollment, potentially diluting the results of the trial. Nearly $13 \%$ of the invasive strategy group had nonobstructive coronary artery disease and did not require revascularization.

Moreover, as noted in the discussion above on quality of life, only a small proportion of patients were severely symptomatic with the potential to experience substantial improvements in quality of life and angina frequency.

Finally, the impact of complete revascularization, mode of revascularization, and invasive functional assessment are yet to be explored in the ISCHEMIA trial.

\section{INVASIVE PHYSIOLOGY ASSESSMENT IN STABLE CORONARY ARTERY DISEASE}

Findings from trials using the instantaneous wave-free ratio (IFR), ${ }^{17,18}$ or fractional flow reserve (FFR $)^{19-21}$ have suggested that these functional measurements of hemodynamic significance may help identify patients for whom revascularization is best suited.

FAME 2. The Fractional Flow Reserve Versus Angiography for Multivessel Evaluation 2 (FAME 2) randomized trial ${ }^{15}$ found 


\section{Abnormal stress test in stable coronary artery disease} with normal left ventricular function

Symptomatic stable angina<smiles></smiles>

Optimal medical therapy (aspirin, 2 antianginals such as nitrates, beta-blocker, calcium channel blocker)

Manage risk factors (smoking cessation, statin, blood pressure control, lifestyle modification, weight loss)

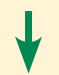

Coronary computed tomography angiography to exclude left main disease

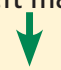

Consider invasive FFR-IFR guided revascularization to improve quality of life and refractory angina despite maximal anginal therapy with at least two potent antianginals with moderate to severe ischemia

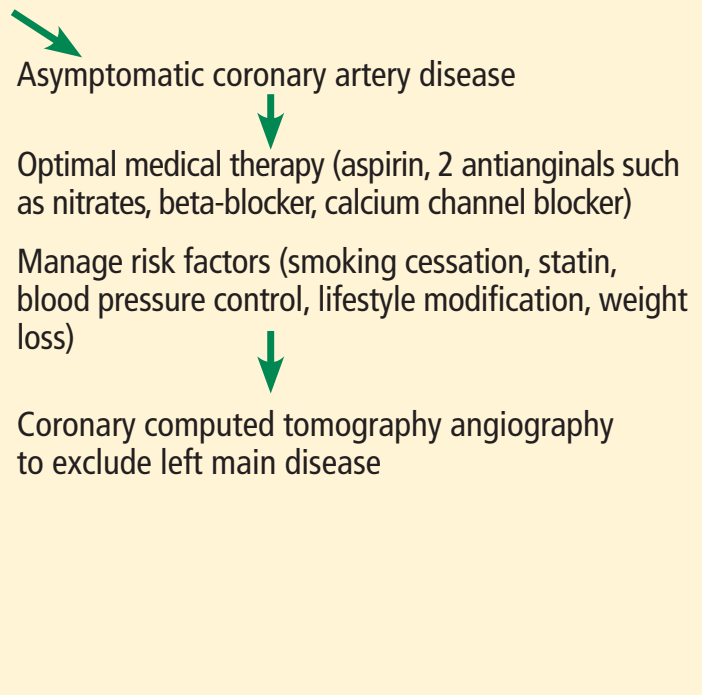

$\mathrm{FFR}=$ fractional flow reserve; IFR = instantaneous wave-free ratio

Figure 1. Algorithm for the management of stable coronary artery disease.

that in patients with stable angina and FFR 0.80 or less, percutaneous coronary intervention reduced the rate of death, nonfatal myocardial infarction, and urgent revascularization at 5 years compared with medical therapy, a benefit driven by a reduction in the rate of urgent revascularization. Patients deferred for revascularization based on a nonsignificant FFR value of more than 0.80 had a good long-term prognosis.

Similar to the ISCHEMIA trial findings, the FAME 2 trial $^{15}$ also demonstrated a trend in which percutaneous coronary intervention was associated with a nearly significant reduction in myocardial infarction at 5 years (HR 0.66, 95\% CI 0.43-1.00) compared with medical therapy.

The RIPCORD trial (Does Routine Pressure Wire Assessment Influence Management Strategy at Coronary Angiography for Diagnosis of Chest Pain? ${ }^{22}$ evaluated the routine use of pressure wire assessment during coronary angiography and demonstrated a drastic difference in decision- making before and after pressure wire assessment.

This finding provided the hypothesis for the RIPCORD 2 trial, ${ }^{23}$ which will evaluate all patients undergoing catheterization for elective indications and urgent stabilized non-ST-elevation acute coronary syndromes with a routine pressure wire. The primary out- come measures are economic and quality of life. This trial will shed more light on FFRguided angiography and steer further trials in this arena targeting hard end points.

\section{ONE SIZE DOES NOT FIT ALL}

The population of the ISCHEMIA trial ${ }^{1-3}$ was heterogeneous and included patients with or without symptoms, assessed with various imaging tests proving ischemia. Importantly, the trial excluded patients with intolerable angina. The results were consistent with those of previous trials, and percutaneous coronary intervention had no survival benefit in patients with stable coronary artery disease without severe left main disease.

Importantly, however, revascularization did improve quality of life and reduce angina frequency in moderately or severely symptomatic patients. Moreover, rates of spontaneous myocardial infarction and hospitalizations for unstable angina were lower in the invasive treatment group. Based on the trial findings, tools can be developed that would predict the possible advantages and disadvantages of each management strategy in individual patients.

The ISCHEMIA trial ${ }^{1-3}$ does not change the indication for revascularization in stable coronary artery disease that has been estab-

\section{Revascul-} arization improved quality of life and reduced angina frequency 
lished so far. Revascularization should be reserved to treat individuals with refractory angina and moderate to severe ischemia to improve the quality of life. The ISCHEMIA trial $^{1-3}$ results do not apply to patients with significant unprotected left main disease, ischemic cardiomyopathy, or acute coronary syndromes.

Figure 1 is a suggested algorithm for managing patients with stable angina. The ISCHEMIA trial provides added reassurance that patients without symptoms with an abnormal stress test can be treated noninvasively once left main disease is excluded.

Stable coronary artery disease needs a patient-centered approach, and one size does not fit all. Management should take into consideration the clinical history, impact on quality of life, risk factors, and the burden of ischemia. A multidisciplinary approach and up-front, frank discussion is necessary regarding an invasive strategy, contemplating the risk and benefits.

\section{REFERENCES}

1. Maron DJ, Hochman JS, Reynolds HR, et al. Initial invasive or conservative strategy for stable coronary disease. N Engl J Med 2020; 382(15):1395-1407. doi:10.1056/NEJMoa1915922

2. ISCHEMIA Trial Research Group; Maron DJ, Hochman JS, O'Brien SM, et al. International Study of Comparative Health Effectiveness with Medical and Invasive Approaches (ISCHEMIA) trial: rationale and design. Am Heart J 2018 Jul; 201:124-135 doi:10.1016/j.ahj.2018.04.011

3. Spertus JA, Jones PG, Maron DJ, et al. Health-status outcomes with invasive or conservative care in coronary disease. N Engl J Med 2020; 382(15):1408-1419. doi:10.1056/NEJMoa1916370

4. Nedelman M. For heart disease, meds may work as well as invasive surgery, major trial shows. CNN. Updated 5:51 pm ET, Sat November 16, 2019. https://www.cnn. com/2019/11/16/health/ischemia-trial-invasive-stableheart-disease-study-bn/index.html

5. Johnson CY. Stents and bypass surgery are no more effective than drugs for stable heart disease, highly anticipated trial results show. Washington Post 2019 Nov 16. https://www.washingtonpost.com/

6. Keeley EC, Boura JA, Grines CL. Primary angioplasty versus intravenous thrombolytic therapy for acute myocardial infarction: a quantitative review of 23 randomised trials. Lancet 2003; 361(9351):13-20. doi:10.1016/S0140-6736(03)12113-7

7. Fox KA, Clayton TC, Damman P, et al. Long-term outcome of a routine versus selective invasive strategy in patients with non-ST-segment elevation acute coronary syndrome a meta-analysis of individual patient data. J Am Coll Cardiol 2010; 55(22):2435-2445.

doi:10.1016/j.jacc.2010.03.007

8. Bittl JA, He Y, Jacobs AK, Yancy CW, Normand SL. Bayes-

\section{KEY POINTS}

- The ISCHEMIA trial indicates that patients who have stable coronary artery disease, an abnormal stress test, and no left main disease can be medically managed safely without invasive treatment.

- Cardiac computed tomography is useful to exclude left main disease.

- The ISCHEMIA trial results are relevant to stable coronary artery disease and do not apply to significant unprotected left main disease, ischemic cardiomyopathy, and acute coronary syndromes.

- Interestingly, rates of spontaneous myocardial infarction and hospitalizations for unstable angina were lower in the invasive treatment group than in the conservative management group. However, further research is necessary to confirm this finding at a large scale.

- Revascularization continues to be beneficial in patients with refractory angina and moderate to severe ischemia to improve the quality of life.

ian methods affirm the use of percutaneous coronary intervention to improve survival in patients with unprotected left main coronary artery disease. Circulation 2013; 127(22):2177-2185 doi:10.1161/CIRCULATIONAHA.112.000646

9. Yusuf S, Zucker D, Peduzzi P, et al. Effect of coronary artery bypass graft surgery on survival: overview of 10-year results from randomised trials by the Coronary Artery Bypass Graft Surgery Trialists Collaboration. Lancet 1994; 344(8922):563-570. doi:10.1016/s0140-6736(94)91963-1

10. Boden WE, O'Rourke RA, Teo KK, Optimal medical therapy with or without $\mathrm{PCl}$ for stable coronary disease. N Engl J Med 2007; 356(15):1503-1516. doi:10.1056/NEJMoa070829

11. Bangalore S, Maron DJ, O'Brien SM. Management of coronary disease in patients with advanced kidney disease. N Engl J Med 2020; 382(17):1608-1618. doi: 10.1056/NEJMoa1915925

12. Prasad A, Gersh BJ, Bertrand ME, et al. Prognostic significance of periprocedural versus spontaneously occurring myocardial infarction after percutaneous coronary intervention in patients with acute coronary syndromes: an analysis from the ACUITY (Acute Catheterization and Urgent Intervention Triage Strategy) trial. J Am Coll Cardiol 2009; 54(5):477-486. doi:10.1016/j.jacc.2009.03.063

13. Bangalore S, Maron DJ, Fleg JL, et al. International Study of Comparative Health Effectiveness with Medical and Invasive Approaches-Chronic Kidney Disease (ISCHEMIACKD): rationale and design. Am Heart J 2018 Nov; 205:42-52. doi:10.1016/j.ahj.2018.07.023

14. Rock CL, Flatt SW, Pakiz B, et al. Weight loss, glycemic control, and cardiovascular disease risk factors in response to differential diet composition in a weight loss program in type 2 diabetes: a randomized controlled trial. Diabetes Care 2014; 37(6):1573-1580. 
doi:10.2337/dc13-2900

15. De Bruyne B, Pijls NH, Kalesan B, et al. Fractional flow reserve-guided $\mathrm{PCl}$ versus medical therapy in stable coronary disease. N Engl J Med 2012; 367(11):991-1001. doi:10.1056/NEJMoa1205361

16. Spertus JA, Jones PG, Maron DJ, et al. Health status after invasive or conservative care in coronary and advanced kidney disease. N Engl J Med 2020; 382(17):1619-1628. doi:10.1056/NEJMoa1916374

17. Gotberg M, Christiansen EH, Gudmundsdottir IJ, et al. Instantaneous wave-free ratio versus fractional flow reserve to guide $\mathrm{PCl}$. N Engl J Med 2017; 376(19):18131823. doi:10.1056/NEJMoa1616540

18. Davies JE, Sen S, Dehbi HM, et al. Use of the instantaneous wave-free ratio or fractional flow reserve in $\mathrm{PCl} . \mathrm{N}$ Engl J Med 2017; 376(19):1824-1834. doi:10.1056/NEJMoa1700445

19. Xaplanteris $\mathbf{P}$, Fournier $\mathbf{S}, \mathrm{Pijls} \mathbf{N H J}$, et al. Five-year outcomes with $\mathrm{PCl}$ guided by fractional flow reserve. $\mathrm{N}$ Engl J Med 2018; 379(3):250-259. doi:10.1056/NEJMoa1803538

20. Nagel E, Greenwood JP, McCann GP, et al. Magnetic resonance perfusion or fractional flow reserve in coronary disease. N Engl J Med 2019; 380(25):2418-2428.

\section{doi:10.1056/NEJMoa1716734}

21. Tonino PA, De Bruyne B, Pijls NH, et al. Fractional flow reserve versus angiography for guiding percutaneous coronary intervention. N Engl J Med 2009; 360(3):213224. doi:10.1056/NEJMoa0807611

22. Curzen N, Rana O, Nicholas Z, et al. Does routine pressure wire assessment influence management strategy at coronary angiography for diagnosis of chest pain?: the RIPCORD study. Circ Cardiovasc Interv 2014; 7(2):248-255. doi:10.1161/CIRCINTERVENTIONS.113.000978

23. Elguindy M, Stables R, Nicholas Z, Kemp I, Curzen N. Design and rationale of the RIPCORD 2 trial (Does Routine Pressure Wire Assessment Influence Management Strategy at Coronary Angiography for Diagnosis of Chest Pain?): a randomized controlled trial to compare routine pressure wire assessment with conventional angiography in the management of patients with coronary artery disease. Circulation Cardiovasc Qual Outcomes 2018; 11(2):e004191. doi:10.1161/CIRCOUTCOMES.117.004191

Address: A. Michael Lincoff, MD, Department of Cardiovascular Medicine, Cleveland Clinic, 9500 Euclid Avenue, Cleveland, OH; LINCOFA@ccf.org 